\title{
Giant panda protection: challenges and hopes
}

\author{
Dongwei Kang ${ }^{1} \cdot$ Junqing $\mathrm{Li}^{1}$
}

Received: 5 November 2018 / Accepted: 6 May 2019 / Published online: 16 May 2019

(C) Springer-Verlag GmbH Germany, part of Springer Nature 2019

\begin{abstract}
In this paper, we discussed some major issues that hinder giant panda protection, such as diverse and mixed threats, habitat fragmentation, as well as the survey method to be improved, and some new protection actions appeared, such as the pilot program for the giant panda national park system and the administration of the giant panda national park was established. These information could provide important information for giant panda protection.
\end{abstract}

Keywords Giant panda $\cdot$ Habitat fragmentation $\cdot$ Threats $\cdot$ Giant panda national park $\cdot$ Ecological civilization

The protection of the giant panda (Ailuropoda melanoleuca) should be a successful demonstration of the harmonious coexistence between humans and nature. According to data released by the former State Forestry Administration of China, from the third to the fourth survey of the giant panda, the number of wild panda individuals increased from 1596 to 1864 , and the area of its habitat increased from 2,304,991 to 2,580,000 $\mathrm{hm}^{2}$ (State Forestry Administration 2006; State Council Information Office of China 2015). While much has been achieved, we believe that some major issues hinder its protection, such as diverse and mixed threats, habitat fragmentation, as well as the survey method to be improved. However, some new protection actions also have appeared, such as the pilot program for the giant panda national park system and the administration of the giant panda national park was established, which brings new hopes to giant panda protection. In this paper, we discussed the challenges and hopes of giant panda protection; we hope it could provide important information for a clearer understanding of the current situation of giant panda protection and promote more targeted protection measures.

The scope and intensity of the third and fourth giant panda surveys were different - the area covered under the third survey was $2,539,789 \mathrm{hm}^{2}$ and the number of sampling lines

Responsible editor: Philippe Garrigues

Junqing Li

lijunqing8100@163.com

1 College of Forestry, Beijing Forestry University, No.35 East-Qinghua Road Haidian District, Beijing 100083, China were 11,174 (State Forestry Administration 2006), whereas, the corresponding figures for the fourth survey were $4,360,000 \mathrm{hm}^{2}$ and 20,513, respectively (State Council Information Office of China 2015). Given this, to better understand the population dynamics and habitat changes of giant pandas, improving the survey method should be considered in future surveys.

Although giant panda protection has received more support, giant panda's habitat has become more fragmented (Kang and Li 2016). A recent study showed that the current extent of giant panda habitat is more fragmented than in the 1980s (Xu et al. 2017). Currently, in terms of the extent of fragmentation, the giant panda's habitat in comparison with its historical distribution (Loucks et al. 2001) may be in a worse state, and therefore, a big challenge for protecting the giant panda is in identifying ways to improve its habitat.

The giant panda and its habitats face several threats, both anthropogenic and natural, such as grazing, road building, herb collection, logging, tourism, hydropower stations, mining, landslides and mud-rock flow, bamboo flowering, and earthquakes (State Forestry Administration 2006; Sichuan Forestry Department 2015; State Council Information Office of China 2015; Shi 2017; Zhou et al. 2017). Among these threats, some are permanent building facilities and some are mobility. Furthermore, some are random events, while others are periodic events. Spatially, these threats can influence at a point scale, linearly, or over a larger area. Temporally, some of these threats have ceased, but their influence is yet to be eliminated. Some threats are current, while others are about to happen, or may happen. Thus, these threats have different attributes across space and time scales, appear alone or together, and bring in their wake, a big challenge to our 
understanding of how they affect the pandas and their habitats, and in identifying measures that should be taken to respond to them.

In fact, in order to protect the giant panda, measures to protect and restore their habitats need to be taken in accordance with the different spatial scales-from specific sites to an entire ecosystem, from landscapes to distribution areas. Furthermore, implementing general strategies alone may be insufficient, because the habitat requirements of giant pandas differ in different areas (Zhang and $\mathrm{Hu}$ 2000; Hu 2001; Wei et al. 2011). As a result, the giant panda protection itself is a complicated mission, with different threats making it even more so.

Notable among the natural threats is bamboo flowering, which is a periodic event. Arrow bamboo flowering occurs every 45 years (Schaller 1993), and the next mass flowering over a large area since the 1970s and 1980s (Editorial Board of China Agriculture Yearbook 1984) is expected to occur soon. Bamboo flowering is not an abnormal phenomenon (Pan et al. 2001) and should not be considered a disaster for pandas. However, under the current condition of more fragmented habitats, some small populations may face a crisis when bamboo flowering occurs because of food shortage and/or constraints faced when attempting to migrate out of their habitats. Given that during the period of arrow bamboo flowering from 1974 to 1976,138 dead panda individuals were found (Editorial Board of China Agriculture Yearbook 1984), it is advisable to be alert to the effects of bamboo flowering.

Climate change may be a potential threat, but its effect has been over-estimated (Wang et al. 2018). Factors such as the uncertainty of future climate, the development of science and technology, and policy adjustments will challenge or even overthrow some previous predictions. Even so, we also support the setting aside of some areas that currently have no panda populations as future panda habitats (Liu 2015), which is not only an important response to mitigate the effects of climate change on the panda but also important for their daily protection.

Given these threats and challenges, China has been taking actions to promote the overall protection of the giant panda. For example, a pilot program for the giant panda national park system was approved, which aims to establish the giant panda national park with a total area of $27,134 \mathrm{~km}^{2}$ (People's Government of Sichuan Province 2017), an area larger than their current habitat area. Furthermore, the administration of the giant panda national park was also established in 2018 . However, the detailed scheme and progress have not yet been made public, resulting in uncertainty surrounding its implementation and in solving the problems of panda protection.

With rapid economic development, China is paying more and more attention to environmental protection. Recently, ecological civilization has been written into the Chinese constitution, an action which will facilitate the harmonious development between humans and nature. To respond to the Chinese government's call to facilitate the ecological civilization, the protection of the giant panda should be well integrated into this national policy, and a matching protection plan should be followed up in time.

Funding information This study was financially supported by the Fundamental Research Funds for the Central Universities (2018ZY36).

\section{References}

Editorial Board of China Agriculture Yearbook (1984) China agriculture yearbook 1984. China Agricultural Press, Beijing

Hu J (2001) Research on the giant panda. Shanghai Scientific and Technological Education Publishing House, Shanghai

Kang D, Li J (2016) Premature downgrade of panda's status. Science 354: 295

Liu J (2015) Promises and perils for the panda. Science 348:642

Loucks CJ, Lü Z, Dinerstein E, Wang H, Olson DM, Zhu C, Wang D (2001) Giant pandas in a changing landscape. Science 294:1465

Pan W, Lü Z, Zhu X, Wang D, Wang H, Long Y, Fu D, Zhou X (2001) A chance for lasting survival. Peking University Press, Beijing

People's Government of Sichuan Province (2017) http://www.sc.gov.cn/ 10462/12771/2017/8/9/10430252.shtml

Schaller GB (1993) The last panda. University of Chicago Press, Chicago

Shi Z (2017) The 4th survey report on giant panda in Gansu province. Gansu Science and Techology Press, Lanzhou

Sichuan Forestry Department (2015) The pandas of Sichuan: the 4th survey report on giant panda in Sichuan province. Sichuan Science and Techology Press, Chengdu

State Council Information Office of China (2015) http://www.scio.gov. $\mathrm{cn} / \mathrm{xwfbh} / \mathrm{gbwxwfbh} / \mathrm{fbh} /$ Document/1395514/1395514.htm

State Forestry Administration (2006) The 3rd national survey report on giant panda in China. Science Press, Beijing

Wang F, Zhao Q, McShea WJ, Songer M, Huang Q, Zhang X, Zhou L (2018) Incorporating biotic interactions reveals potential climate tolerance of giant pandas. Conserv Lett 11:e12592

Wei F, Zhang Z, Hu J (2011) Research advances and perspectives on the ecology of wild giant pandas. Acta Theriol Sin 31:412-421

Xu W, Viña A, Kong L, Pimm SL, Zhang J, Yang W, Xiao Y, Zhang L, Chen X, Liu J, Ouyang Z (2017) Reassessing the conservation status of the giant panda using remote sensing. Nat Ecol Evol 1:16351638

Zhang Z, Hu J (2000) Studies on habitat selection by giant pandas. J Sichuan Teachers Coll (Nat Sci) 21:18-21

Zhou L, Zhang X, Jiu Q, Meng X (2017) The pandas of Qinling: the 4th survey report on giant panda in Shaanxi province. Shaanxi science and Techology press, Xi'an

Publisher's note Springer Nature remains neutral with regard to jurisdictional claims in published maps and institutional affiliations. 